\title{
Leukotriene B4 receptor-2 contributes to KRAS-driven lung tumor formation by promoting interleukin-6-mediated inflammation
}

\author{
Jae-Hyun Jang ${ }^{1,5}$, Donghwan Park ${ }^{1,5}$, Guen-soo Park ${ }^{1,5}$, Dong-Wook Kwak', Jaeln Park', Dae-Yeul Yu ${ }^{2}$, Hye Jin You (iD ${ }^{3}$ and \\ Jae-Hong Kim iD ${ }^{4 \times}$
}

(C) The Author(s) 2021

\begin{abstract}
Although lung cancer is the leading cause of cancer-related deaths worldwide and KRAS is the most frequently mutated oncogene in lung cancer cases, the mechanism by which KRAS mutation drives lung cancer has not been fully elucidated. Here, we report that the expression levels of leukotriene $B_{4}$ receptor-2 (BLT2) and its ligand-producing enzymes (5-LOX, 12-LOX) were highly increased by mutant KRAS and that BLT2 or 5-/12-LOX blockade attenuated KRAS-driven lung cell proliferation and production of interleukin6 (IL-6), a principal proinflammatory mediator of lung cancer development. Next, we explored the roles of BLT2 and 5-/12-LOX in transgenic mice with lung-specific expression of mutant KRAS $\left(\mathrm{Kras}^{\mathrm{G}}{ }^{12 \mathrm{D}}\right)$ and observed that BLT2 or 5-/12-LOX inhibition decreased IL-6 production and tumor formation. To further determine whether BLT2 is involved in KRAS-driven lung tumor formation, we established a Kras ${ }^{\mathrm{G} 12 \mathrm{D}} / \mathrm{BLT} 2-\mathrm{KO}$ double-mutant mouse model. In the double-mutant mice, we observed significantly suppressed IL6 production and lung tumor formation. Additionally, we observed high BLT2 expression in tissue samples from patients with KrasG12D-expressing lung adenocarcinoma, supporting the contributory role of BLT2 in KRAS-driven human lung cancer. Collectively, our results suggest that BLT2 is a potential contributor to KRAS-driven lung cancer and identify an attractive therapeutic target for KRAS-driven lung cancer.
\end{abstract}

Experimental \& Molecular Medicine (2021) 53:1559-1568; https://doi.org/10.1038/s12276-021-00682-z

\section{INTRODUCTION}

Lung cancer is the leading cause of cancer-related deaths among both men and women, with more than 1.5 million deaths per year worldwide ${ }^{1}$. Epidermal growth factor receptor (EGFR) mutations are the second most common cause of lung cancer, and KRAS is the most frequently mutated oncogene in lung cancer cases ${ }^{2}$. Despite several notable studies ${ }^{3,4}$, mutant KRAS is difficult to target with small molecules due to its undruggable structure ${ }^{5}$. Because of these limitations, alternative approaches involving lung inflammatory pathways have been explored ${ }^{6}$. Interleukin- 6 (IL-6) is a proinflammatory cytokine that has been implicated in the progression of lung cancers, including KRAS-driven lung cancer ${ }^{7}$. IL- 6 contributes to lung cancer progression by triggering pro-inflammatory pathways that enhance cancer cell proliferation; thus, IL- 6 is considered a marker of poor prognosis in lung cancer $^{8-10}$. Unfortunately, IL-6 blockade has limited effectiveness in lung cancer patients ${ }^{1,12}$, and therefore, alternative molecular targets are required.

Leukotriene $B_{4} \quad\left(L_{T} B_{4}\right)$ is a potent proinflammatory lipid mediator that binds to the cell surface $\mathrm{G}$ protein-coupled receptors $\mathrm{LTB}_{4}$ receptor-1 (BLT1) and $B L T 2^{13}$. BLT1 is expressed mainly on peripheral leukocytes, while BLT2 is minimally expressed under normal conditions but overexpressed in proinflammatory environments ${ }^{14-16}$. Moreover, recent reports have indicated that the BLT2 signaling cascade is associated with lung inflammatory diseases such as asthma ${ }^{17,18}$. Additionally, BLT2 and its ligand-producing enzymes 5-lipoxygenase (5-LOX) and 12lipoxygenase (12-LOX), which convert arachidonic acid into the BLT2 ligands $\mathrm{LTB}_{4}$ and 12(S)-hydroxy-5Z,8Z,10E,14Z-eicosatetraenoic acid (12(S)-HETE), respectively ${ }^{19,20}$, play critical roles in the production of proinflammatory cytokines in lung airway disease in the context of asthma ${ }^{21}$. Despite the suggested role of the BLT2 cascade in lung inflammation, little is known about its contribution to the progression of lung cancer, especially KRAS-mutant lung cancer.

To this end, in this study, we investigated whether BLT2 contributes to mutant KRAS-driven lung cancer progression. We found that the BLT2 cascade lies downstream of mutant KRAS and contributes to mutant KRAS-driven lung cancer cell proliferation and IL- 6 production. Additionally, we revealed that the inhibition of BLT2 decreases lung tumor formation, IL-6 production, and inflammatory phenotypes in the lung. Furthermore, Blt2 knockout (KO) in a mouse model of KRAS-driven lung cancer was associated with strong suppression of lung tumor formation and IL-6 production. Moreover,

\footnotetext{
${ }^{1}$ Department of Biotechnology, College of Life Sciences and Biotechnology, Korea University, Seoul 02841, Republic of Korea. ${ }^{2}$ Disease Model Research Laboratory, Genome Editing Research Center, Korea Research Institute of Bioscience and Biotechnology (KRIBB), Daejeon 34141, Republic of Korea. ${ }^{3}$ Division of Translational Science, National Cancer Center, Goyang 10408, Republic of Korea. ${ }^{4}$ Division of Life Sciences, College of Life Sciences, Korea University, Seoul 02841, Republic of Korea. ${ }^{5}$ These authors contributed equally: Jae-Hyun Jang, Donghwan Park, Guen-soo Park. ${ }^{凶}$ email: jhongkim@korea.ac.kr
} 
Table 1. Information on the lung tissues used in the study.

\begin{tabular}{|c|c|c|c|c|c|c|c|c|}
\hline & Age & Sex & $\begin{array}{l}\text { Organ/ } \\
\text { anatomic site }\end{array}$ & $\begin{array}{l}\text { Pathological } \\
\text { diagnosis }\end{array}$ & Grade & Type & $\begin{array}{l}\text { KrasG12D-positive } \\
\text { area (\%) }\end{array}$ & $\begin{array}{l}\text { BLT2-positive } \\
\text { area (\%) }\end{array}$ \\
\hline Fig. $5 b$ & 68 & Male & Lung & Adenocarcinoma & 2 & Malignant & 8.1 & 7.5 \\
\hline Fig. 5d (left) & 35 & Male & Lung & Normal & & & 0 & 0 \\
\hline Fig. $5 d$ (right) & 55 & Male & Lung & Adenocarcinoma & 2 & Malignant & 9.2 & 8.1 \\
\hline
\end{tabular}

immunohistochemical (IHC) and immunofluorescence (IF) analysis revealed substantial BLT2 expression in tissue samples from lung adenocarcinoma patients with the KrasG12D mutation. Thus, we identified BLT2 as a potential contributor to KRAS-driven lung cancer, and our results may facilitate the development of strategies against KRAS-mutant lung cancer.

\section{MATERIALS AND METHODS \\ Chemicals and plasmids}

RPMI 1640 medium and DMEM were purchased from Corning, Inc. (Corning, NY, USA). Fetal bovine serum (FBS) was purchased from HyClone Laboratories, Inc. (Logan, UT, USA). MK886, baicalein, and LY255283 were purchased from Cayman Chemical (Ann Arbor, Ml, USA). SB203580 was purchased from Calbiochem (San Diego, CA, USA). Dimethyl sulfoxide (DMSO) was purchased from Sigma-Aldrich (St. Louis, MO, USA). The pCGN vectors were purchased from Invitrogen (Carlsbad, CA, USA). Antibodies against BLT2 were obtained from Enzo Life Sciences, Inc. (Farmingdale, NY, USA); antibodies against 5-LOX, $\beta$-actin, phosphorylated cytosolic phospholipase $A_{2}\left(p-c P L A_{2}\right)$, and $p$-IKBa were obtained from Cell Signaling Technology (Danvers, MA, USA), and antibodies against CPLA 2 and 12-LOX were obtained from Santa Cruz Biotechnology, Inc. (Dallas, TX, USA). All other chemicals were obtained from standard sources and were of molecular biology grade or higher.

\section{Cell culture and transfection}

BEAS-2B, A549, and SK-LU-1 cells were obtained from the Korean Cell Line Bank (KCLB, 10061). A549 and SK-LU-1 cells were grown in DMEM supplemented with $10 \% \mathrm{FBS}$ and antibiotic-antimycotic solution (Gibco; El Paso, TX, USA) at $37^{\circ} \mathrm{C}$ in a $5 \% \mathrm{CO}_{2}$ humidified atmosphere. BEAS-2B cells were grown in an RPMI medium under the same conditions. For transient transfection, cells were seeded at $2 \times 10^{5}$ cells per $60-\mathrm{mm}$ plate and incubated for $24 \mathrm{~h}$. Then, DNA-lipid complexes were formed with $4 \mu \mathrm{l}$ of Lipofectamine (Invitrogen) and $2 \mu \mathrm{g}$ of DNA and added to each dish for $18 \mathrm{~h}$. Empty vector DNA was used to equalize the total quantities of transfected DNA between each experiment.

\section{RNA interference (siRNA)}

KRAS-specific and control (scrambled) siRNAs were purchased from Bioneer (Daejeon, Korea). The siRNAs were introduced into cells by transfection as described above for $18 \mathrm{~h}$ in Opti-MEM solution (Invitrogen) using Oligofectamine reagents (Invitrogen).

\section{Reverse transcription (RT)-PCR}

Total RNA was extracted from cells or mouse lung tissue using the easyBLUE $^{\mathrm{TM}}$ Total RNA Extraction Kit (Intron, Gyeonggi-do, Korea). Two micrograms of RNA were subjected to RT with M-MLV reverse transcriptase (Beams Bio, Gyeonggi-do, Korea) followed by semiquantitative PCR with a PCR PreMix Kit (Intron). The primer sequences are provided in Supplementary Table 1. The final PCR products were resolved on a $1.5 \%$ agarose gel containing ethidium bromide and visualized under UV light.

\section{Western blotting}

Cells were collected and lysed with buffer containing $40 \mathrm{mM}$ Tris- $\mathrm{HCl}(\mathrm{pH}$ 8.0), $120 \mathrm{mM} \mathrm{NaCl}, 0.1 \%$ Nonidet $-\mathrm{P} 40,100 \mathrm{mM}$ phenylmethylsulfonyl fluoride, $1 \mathrm{mM}$ sodium orthovanadate, $2 \mathrm{~g} / \mathrm{ml}$ leupeptin, $2 \mathrm{~g} / \mathrm{ml}$ aprotinin, and phosSTOP cocktail (Roche, Basal, Switzerland). Mouse lung tissues were homogenized with buffer containing $100 \mathrm{mM}$ Tris- $\mathrm{HCl}(\mathrm{pH} 7.4)$, $150 \mathrm{mM} \mathrm{NaCl}, 1 \mathrm{mM}$ EGTA, $1 \mathrm{mM}$ EDTA, 1\% Triton X-100, 0.5\% sodium deoxycholate, and proteinase inhibitor cocktail. Proteins were separated by sodium dodecyl sulfate-polyacrylamide gel electrophoresis and transferred to a polyvinylidene fluoride membrane. The membrane was blocked with $5 \%$ nonfat dry milk in Tris-buffered saline for $1 \mathrm{~h}$ and incubated with primary antibodies for $1 \mathrm{~h}$ at room temperature. The membrane was washed for $1 \mathrm{~h}$, and blots were incubated with a peroxidase-conjugated secondary antibody for $1 \mathrm{~h}$ and then washed for $1 \mathrm{~h}$. The bands were visualized by enhanced chemiluminescence (Amersham Biosciences, UK).

\section{Cell counting}

Cells were plated at a density of $5.0 \times 10^{4}$ cells per well in 24-well plates and incubated in medium containing $10 \%$ FBS for $24 \mathrm{~h}$. Then, the cells were incubated with the indicated inhibitors or DMSO in a medium containing $2 \%$ FBS for 24 or $48 \mathrm{~h}$. For measurement of cell proliferation, cells were trypsinized and counted with a hemocytometer using the trypan blue exclusion method.

\section{Mouse models and animal care}

$\mathrm{Kras}^{\mathrm{G} 12 \mathrm{D}}$ mice were kindly provided by Professor Dae-Yeol $\mathrm{Yu}$, who generated these mice via DNA microinjection into B6D2F1 strain (BDF-1) mouse embryos as previously described ${ }^{22}$; a KrasG12D gene expression vector containing the hSP-C promoter was used to drive lung-specific expression of the KrasG12D gene. BLT2 KO mice were constructed as previously described $d^{23}$. The mouse room was maintained at a temperature of $22 \pm 1{ }^{\circ} \mathrm{C}$ and a humidity of $50 \pm 10 \%$ with a 12 -h light/dark cycle. All animal procedures were conducted in accordance with the guidelines of the Korea University Institutional Animal Care \& Use Committee (KUIACUC 2019-0056).

\section{Inhibitor treatment of the KrasG12D mutant mice}

Beginning at 9 weeks of age, the Kras ${ }^{\mathrm{G} 12 \mathrm{D}}$ mice and control littermates were injected intraperitoneally (i.p.) with $0.5 \mathrm{mg} / \mathrm{kg}$ MK886, $5 \mathrm{mg} / \mathrm{kg}$ baicalein, or $2.5 \mathrm{mg} / \mathrm{kg} \mathrm{LY} 255283$ once weekly for 12 weeks. At the experimental endpoint ( 21 weeks of age), five mice from each group were sacrificed and used for analysis. No experimental mice were excluded from any of these analyses.

\section{Generation of Kras ${ }^{\mathrm{G} 12 \mathrm{D}} / \mathrm{BLT} 2 \mathrm{KO}$ mice}

For the construction of the Kras ${ }^{\mathrm{G} 12 \mathrm{D}} / \mathrm{BLT} 2 \mathrm{KO}$ mouse model, Blt2 KO mice on the C57BL/6 background were mated with wild-type (WT) BDF-1 mice to at least the F7 generation. After the acquisition of the BDF-1 strain of

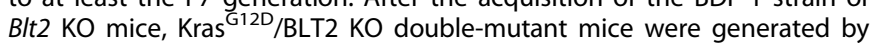
crossing KrasG12D mice with BLT2 KO mice (Supplementary Fig. 1). We used the primers shown in Supplementary Table 1 for genotyping.

\section{Lung histology and analysis of cells in bronchoalveolar lavage fluid (BALF)}

Lung section ( $5 \mu \mathrm{m}$ thickness) were mounted on Superfrost Plus glass slides (Fisher Scientific, Pittsburgh, PA, USA), deparaffinized, and stained with hematoxylin and eosin (H\&E). Inflammatory cells in BALF were collected by centrifugation $(1000 \times g$ for $3 \mathrm{~min}$ ) and washed with PBS. Next, BALF cells were fixed on glass slides and stained with H\&E. Images were acquired using a BX51 microscope (Olympus, Tokyo, Japan) equipped with a DP71 digital camera (Olympus).

\section{Enzyme-linked immunosorbent assay (ELISA)}

Human $\mathrm{LTB}_{4}$ and $12(\mathrm{~S})$-HETE levels were measured using commercially available ELISA kits (Enzo Life Sciences, Inc.), and IL-6 levels were measured using a commercially available ELISA kit (BD Biosciences; Bedford, MA, USA). The $\mathrm{LTB}_{4}, 12(\mathrm{~S})$-HETE, and IL-6 concentrations were measured according to the manufacturers' instructions. 


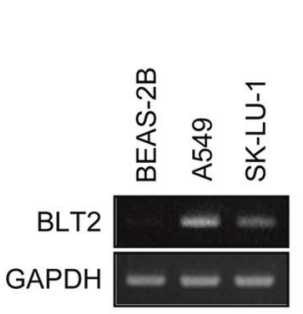

b

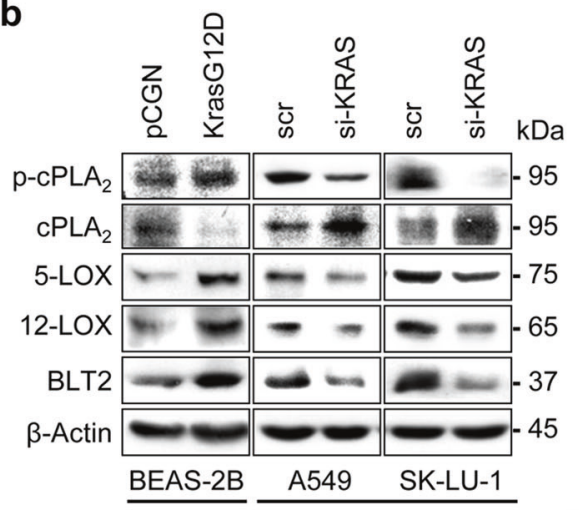

C

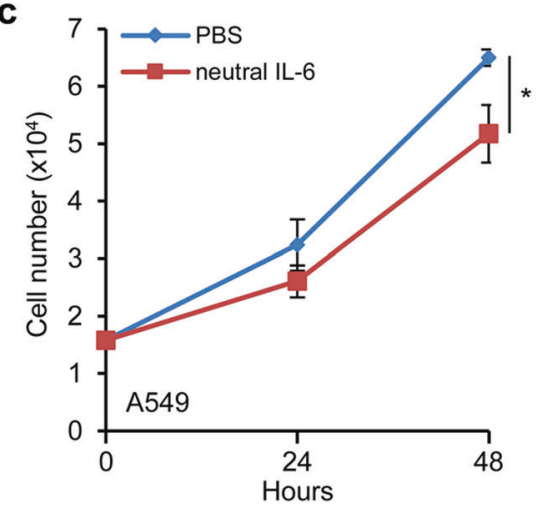

d

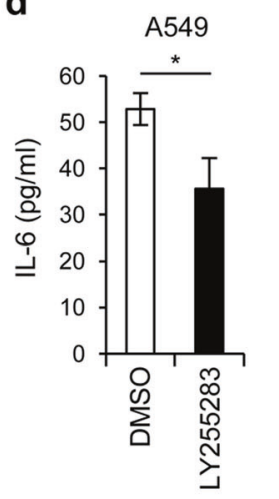

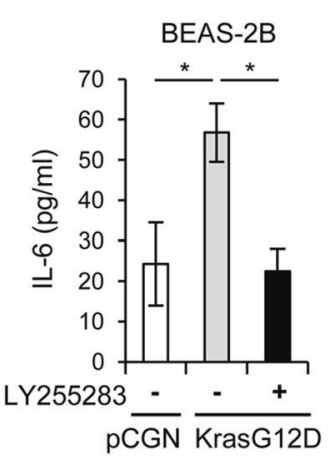

f

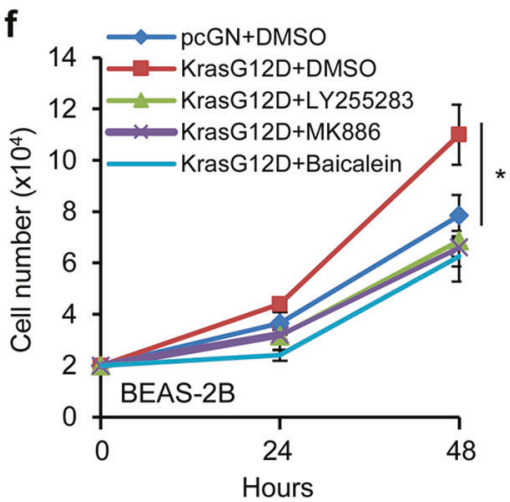

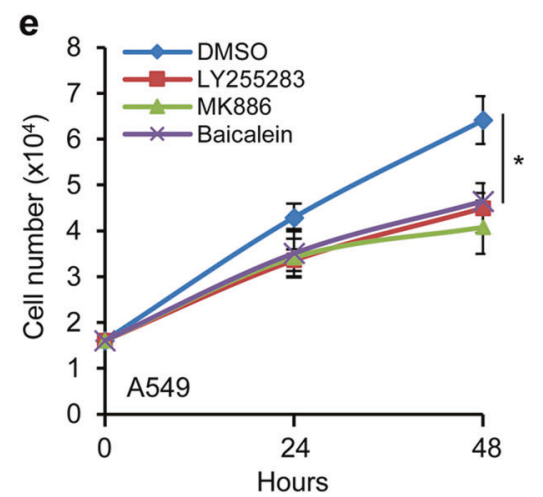

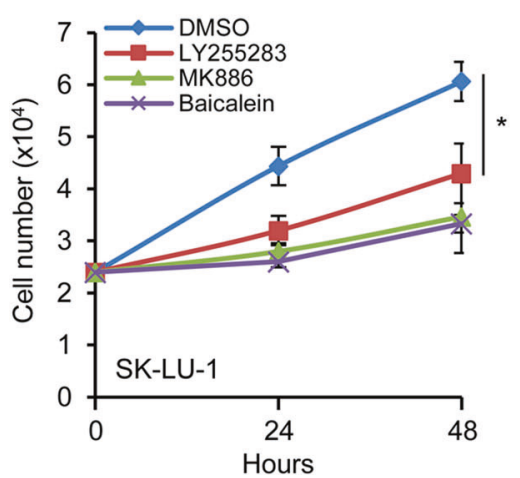

Fig. 1 The BLT2 cascade lies downstream of mutant KRAS in lung cancer cells. a BLT2 mRNA expression and 5-/12-LOX protein expression in BEAS-2B normal lung cells and in A549 and SK-LU-1 KRAS-mutant lung cancer cells. The results are representative of three independent experiments with similar results. b Levels of BLT2 cascade proteins in cell lysates of the indicated cell lines. KrasG12D overexpression was induced in BEAS-2B cells by transfection with a KrasG12D expression plasmid. KRAS was knocked down in A549 and SK-LU-1 cells using siRNAKRAS. Scr scrambled siRNA control. The western blot results are representative of three independent experiments with similar results. Band intensities were quantified using ImageJ and are expressed as the fold change relative to the control value. c A549 cells were treated with an anti-IL-6 neutralizing antibody $(100 \mathrm{ng} / \mathrm{ml})$. Cells were then counted with a hemocytometer. The data are presented as the mean \pm SD values of three independent experiments. d ELISAs of IL-6 production in cell culture supernatants. A549 cells (left) were treated with the BLT2-specific inhibitor LY255283 $(10 \mu \mathrm{M})$. BEAS-2B cells (right) were transfected with the KrasG12D expression plasmid. Twenty-four hours after transfection, the cells were exposed to LY255283 $(10 \mu \mathrm{M})$. The data are presented as the mean \pm SD values of three independent experiments. e A549 (left) and SK-LU-1 cells (right) were treated with inhibitors of BLT2, 5-LOX, and 12-LOX (10 $\mu \mathrm{M}$ LY255283, $5 \mu \mathrm{M}$ MK886, and 800 nM baicalein, respectively) and then counted. The data are presented as the mean \pm SD values of three independent experiments. $f$ BEAS-2B cells were transfected with the KrasG12D expression plasmid. Twenty-four hours after transfection, the cells were exposed to the indicated inhibitor and then counted. Cell proliferation data were statistically analyzed at $48 \mathrm{~h}$ and compared between the indicated inhibitor groups and the DMSO control group. The data are presented as the mean \pm SD values of three independent experiments. The data were analyzed using an unpaired two-tailed Student's $t$-test. ${ }^{*} p<0.05$.

\section{IHC and IF analyses}

Paraffin-embedded lung tissues from lung adenocarcinoma patients were purchased from US Biomax (Derwood, MD, USA) and subjected to IHC and IF analyses. The patient samples were deparaffinized, and antigen retrieval was performed by microwaving the sample in a $0.01 \mathrm{M}$ sodium citrate buffer. The samples were incubated with primary antibodies against KrasG12D (ab221163, Abcam, Cambridge, UK; $7.9 \mu \mathrm{g} / \mathrm{ml}$ ) and BLT2 (PA533911, Thermo Scientific, Waltham, MA, USA; $12 \mu \mathrm{g} / \mathrm{ml}$ ). Endogenous 


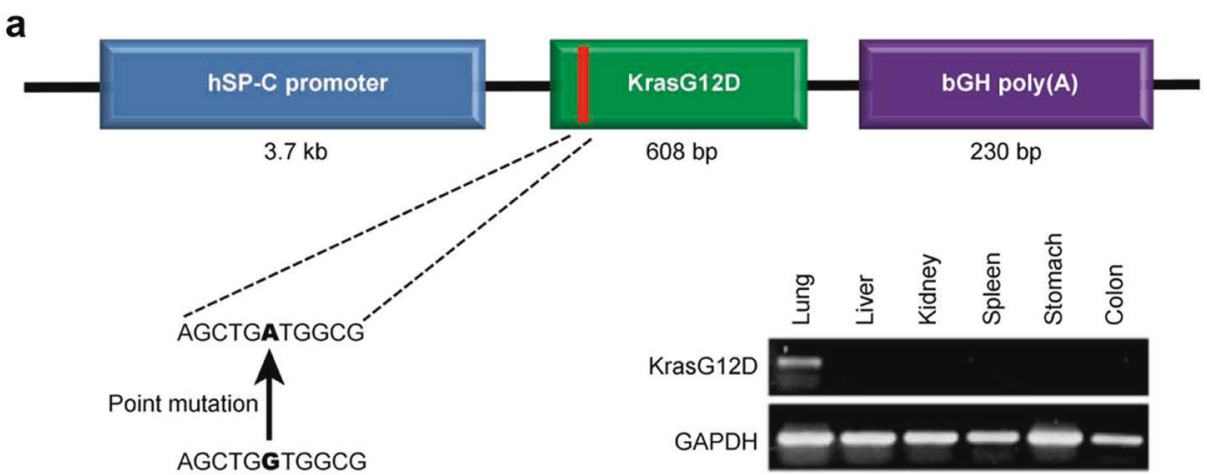

b

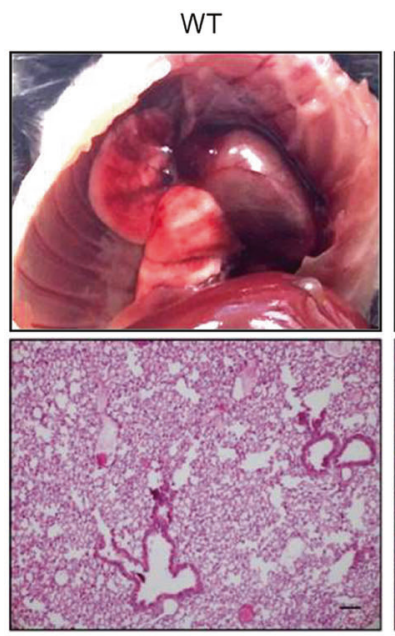

KrasG12D TG

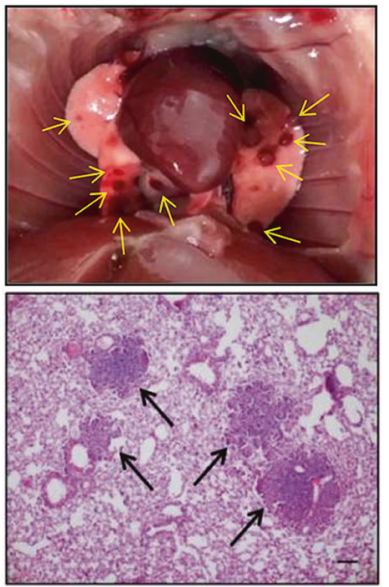

C
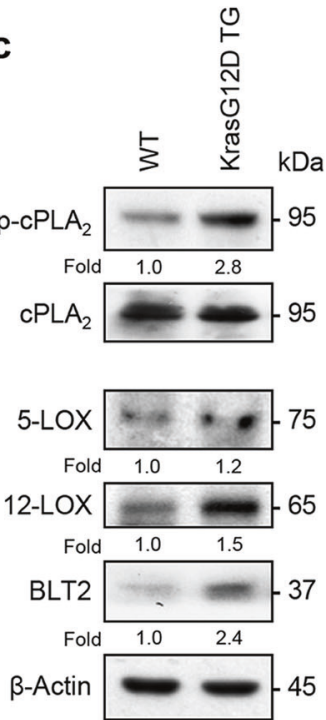

Fig. 2 The levels of 5-/12-LOX and BLT2 are increased in the Kras ${ }^{\text {G12D }}$ mice. a Strategy with the promoter and transgene was used to establish lung-specific KrasG12D expression in mice. b Representative images of lung lesions and H\&E-stained lung sections from the indicated groups of 8-week-old mice. The arrows indicate lung tumors. Scale bars, $50 \mu \mathrm{m}$. c The protein levels of $\mathrm{p}$-cPLA $2,5-/ 12-\mathrm{LOX}$, and BLT2 in the lung tissue extracts of the Kras ${ }^{\mathrm{G} 12 \mathrm{D}}$ mice were analyzed by western blotting. Total $\mathrm{CPLA}$ and $\beta$-actin were used as controls. Three mice were tested for analysis. Similar results were obtained, and representative data are shown. Band intensities were quantified using ImageJ and are expressed as a fold of the control value (cPLA $A_{2}$ as a control for $\mathrm{p}$-CPLA $A_{2} ; \beta$-actin as a control for 5-LOX, 12-LOX, and BLT2).

peroxidase activity was blocked with $0.3 \% \mathrm{H}_{2} \mathrm{O}_{2}$. Samples were stained using the avidin-biotin-peroxidase complex method with an $A B C$ staining kit (Thermo Scientific) and then counterstained with hematoxylin. The stained sections of the lung were observed using a BX51 microscope (Olympus, Tokyo, Japan) equipped with a DP71 digital camera (Olympus). For IF staining of KrasG12D and BLT2, primary antibodies against KrasG12D (ab221163, Abcam) and BLT2 (ADI-905-794-100, Enzo Life Sciences, Farmingdale, NY, USA) were conjugated with FITC and PE, respectively, using a conjugation kit (ab188285, ab102918, Abcam). The patient samples were deparaffinized, rehydrated, and blocked with buffer (PBS containing $1 \% \mathrm{BSA}$ ) for $1 \mathrm{~h}$ at RT. The lung tissues were then incubated overnight at $4{ }^{\circ} \mathrm{C}$ with FITC-conjugated KrasG12D and PE-conjugated BLT2 antibodies. After three washes in PBS, the slides were incubated with DAPI (SigmaAldrich, St. Louis, MO, USA). The slides were washed in PBS, mounted, and observed under a confocal laser scanning microscope (LSM 700, Carl Zeiss, Oberkochen, Germany). Specific information on the patient-derived lung adenocarcinoma tissues is provided in Table 1.

\section{Comparison of BLT2 gene amplification in the KRAS mutant and KRAS wild type samples}

For analysis of BLT2 gene amplification in KRAS mutant lung cancer patients, we analyzed the Pan-Lung Cancer database (TCGA, Nat Genet 2016) cohort of 1144 lung cancer patients via cBioPortal based on the KRAS mutation and BLT2 gene amplification (http://www.cbioportal.org/ study/summary?id=nsclc_tcga_broad_2016).

\section{Statistical analysis}

All quantitative data are shown as the mean $\pm S D$ of three independent experiments. Statistical comparisons between experimental groups were performed using Student's t-test. $p<0.05$ was considered significant. $p<$ $0.05, p<0.01$, and $p<0.001$ are designated by ${ }^{*},{ }^{* *}$, and ${ }^{* * *}$, respectively.

\section{RESULTS}

BLT2, 5-LOX, and 12-LOX expression is significantly increased in KRAS-mutant lung cancer cells

We began our study of the role of BLT2 in KRAS-mutant lung cancer by examining the basal expression levels of BLT2, 5-LOX, and 12-LOX in KRAS-mutant lung cancer cells. The expression levels of BLT2, 5-LOX, and 12-LOX were increased (Fig. 1a) in two human lung cancer cell lines with activating KRAS mutations (A549 with the KrasG12S mutation, and SK-LU-1 with the KrasG12D mutation) compared with a noncancerous control cell line (BEAS-2B, human bronchial epithelial cell line).

To determine whether mutant KRAS expression is associated with the expression of the BLT2 cascade, we transfected BEAS-2B cells with a mutant KRAS expression plasmid (pCGN-Kras ${ }^{G 12 D}$ ). The KrasG12D-expressing BEAS-2B cells showed increased protein levels of 5-LOX, 12-LOX, and BLT2 compared with the control 
a

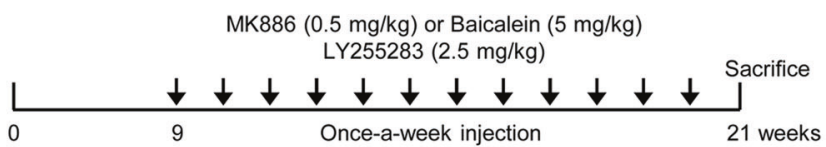

b

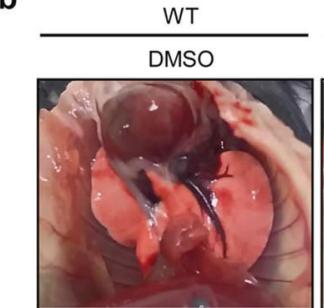

C

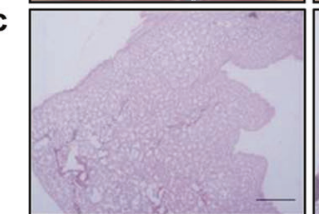

d

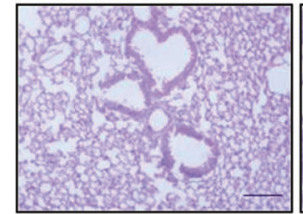

g
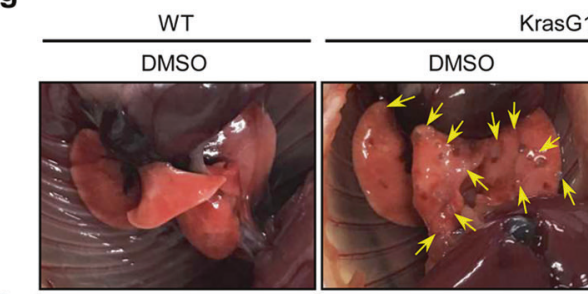

h

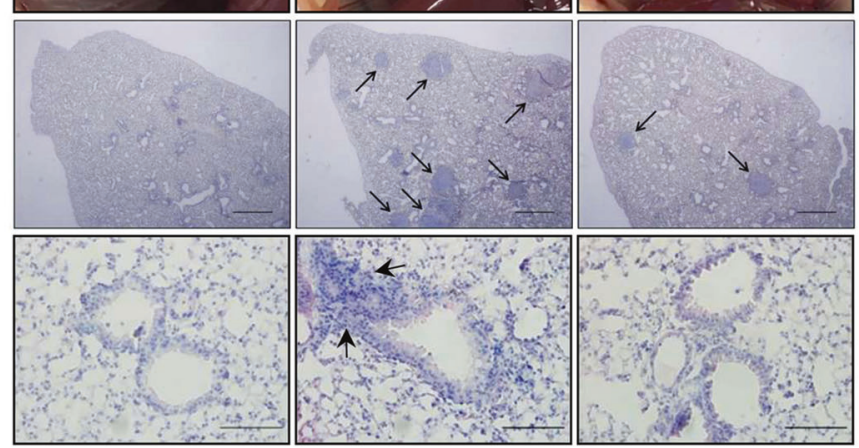

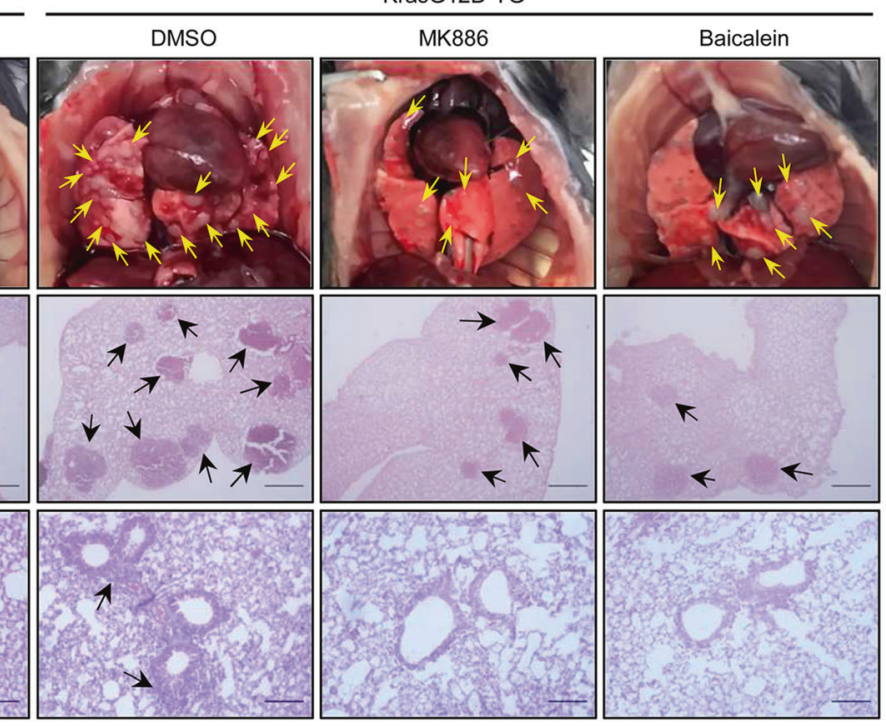

KrasG12D TG

j

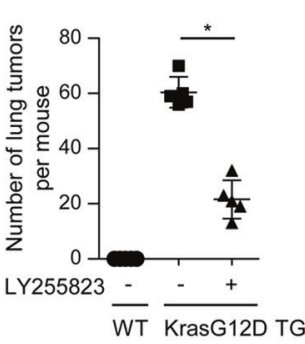

I

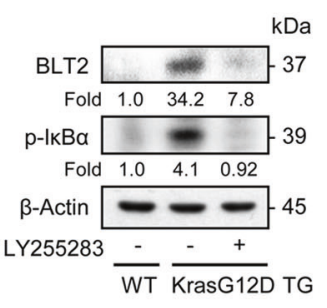

e

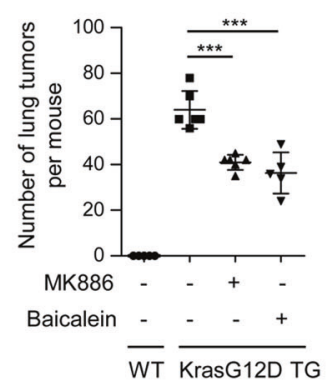

f

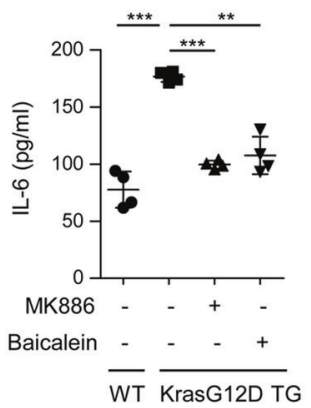

k

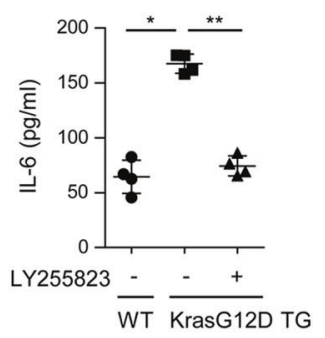

m

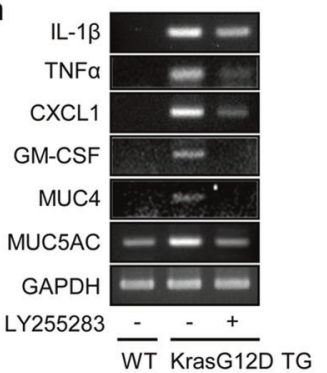

Fig. 3 Inhibition of the 5-/12-LOX-BLT2 cascade suppresses KrasG12D-driven lung tumor formation and IL-6 production in the Kras ${ }^{\text {G12D }}$ mice. a Experimental schedule for BLT2 cascade inhibition in the Kras ${ }^{\mathrm{G} 12 \mathrm{D}}$ mice. $\mathbf{b}, \mathbf{g}$ Representative images of lung lesions. $\mathbf{c}$, $\mathbf{h} \mathrm{H} \& \mathrm{E}$ staining of lung tissues. Scale bars, $500 \mu \mathrm{m}$. The arrows indicate lung tumors. d, i Airway cellular infiltration in the indicated experimental groups. Scale bars, 100 and $200 \mu \mathrm{m}$, respectively. The arrows indicate infiltrated cells. e, $\mathbf{j}$ The number of lung tumors in each mouse was counted. The data are presented as the mean $\pm \mathrm{SD} ; n=5 .{ }^{*} p<0.05$ and ${ }^{* * *} p<0.001$ according to Student's $t$-test. f, $\mathbf{k}$ Lung tissue extract was collected for IL- 6 measurement by ELISAs. The data are shown as the mean \pm SD; $n=5$. ${ }^{*} p<0.05,{ }^{* *} p<0.01$ and ${ }^{* * *} p<0.001$ according to Student's $t$-test. I The expression level of BLT2 and phosphorylation level of $\mid \kappa B \alpha$ in lung tissue extracts were assessed by western blotting. The results are representative of $n=3$ independent experiments. Band intensities were quantified using ImageJ and are expressed as the fold change relative to the control value. $\mathbf{m}$ Total RNA was isolated from mouse lungs, and semiquantitative PCR analysis of the indicated mRNAs was conducted. The data are representative of three independent experiments with similar results.

vector-expressing BEAS-2B cells (Fig. 1b). Similarly, the levels of phosphorylated $c P L A_{2}\left(p-c P L A_{2}\right)$, an activated form of $c P L A_{2}$ that catalyzes the production of arachidonic acid, a precursor molecule for eicosanoid synthesis ${ }^{24}$, were strongly increased by mutant
KRAS expression (Fig. 1b). In addition, we used a targeted small interfering RNA to knock down KRAS (si-KRAS) in A549 and SK-LU1 cells and found that the levels of $\mathrm{p}-\mathrm{CPLA}_{2}, 5-\mathrm{LOX}, 12-\mathrm{LOX}$, and BLT2 were substantially reduced compared with those of the 


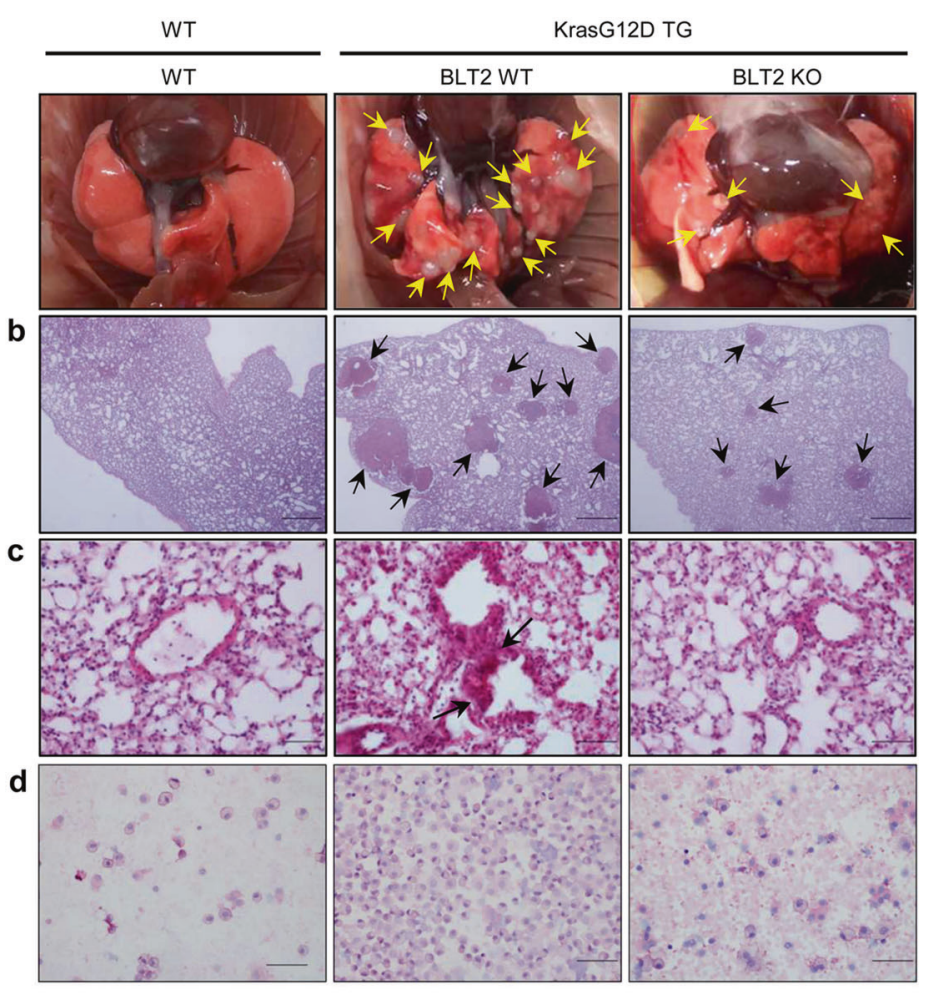

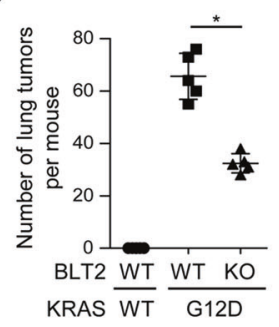

g

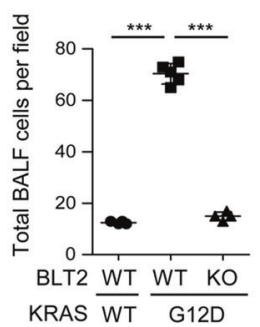

i

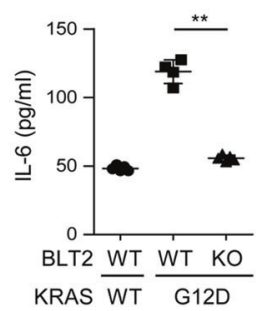

f

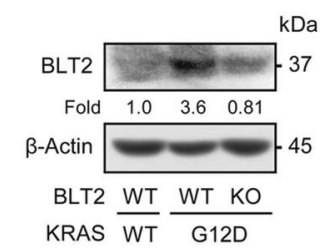

h

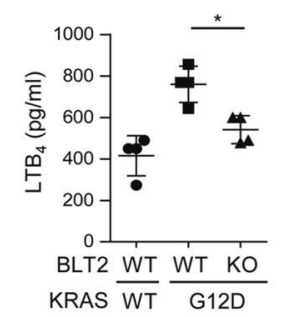

j

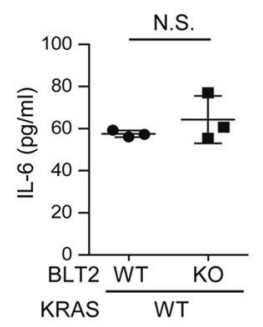

Fig. 4 BLT2 knockout suppresses KrasG12D-driven lung tumor formation and IL-6 production. a Representative images of lung lesions and b H\&E staining of lung tissue. Scale bars, $500 \mu \mathrm{m}$. c Airway cellular infiltration in the indicated experimental groups. The arrows indicate infiltrated cells. Scale bars, $100 \mu \mathrm{m}$. d Representative images of BALF cytospin preparations isolated from each group. The results are representative of five independent experiments with similar results. e The number of lung tumors in the mice was counted. The data are presented as the mean $\pm \mathrm{SD} ; n=5$. ${ }^{*} p<0.05$ according to Student's $t$-test. $\mathrm{f}$ BLT2 protein levels in lung lysates as measured by western blotting. The results are representative of three independent experiments with similar results. Band intensities were quantified using ImageJ and are expressed as the fold change relative to the control value. $\mathbf{g}$ The average number of BALF cells per field of view was determined. The results are presented as the mean \pm SD values; $n=5$. $^{* * *} p<0.001$ with Student's $t$-test. Lung tissue extracts were collected to analyze (h) LTB ${ }_{4}$ and (i, j) IL- 6 by ELISAs. The data are presented as the mean \pm SD; $n=5$. ${ }^{*} p<0.05$ and ${ }^{* *} p<0.01$ according to Student's $t$-test. N.S. not significant.

scrambled siRNA control (Fig. 1b). Collectively, these results suggest that mutant KRAS regulates the expression of the BLT2 cascade in lung cancer cells.

\section{BLT2 or 5-/12-LOX inhibition decreases IL-6 production and cell proliferation}

Recent studies have demonstrated that IL- 6 is critical for lung tumor progression and thus acts as a key driver of lung cancer ${ }^{25-27}$. Accordingly, we observed that treatment of A549 lung cancer cells with an anti-IL-6 neutralizing antibody significantly reduced cell proliferation, as indicated by the $20.4 \%$ decrease in the number of cells in the cell count assays (Fig. 1c), suggesting that IL-6 production is associated with KRAS-mutant lung cancer cell proliferation. Next, to determine whether BLT2 mediates IL- 6 production, we evaluated the effect of treatment with a BLT2specific antagonist (LY255283) on IL-6 levels in A549 cell culture supernatants by ELISAs; this treatment strongly reduced the IL- 6 levels (Fig. 1d, left panel). Additionally, the levels of IL-6 in the BEAS-2B cells transfected with the KrasG12D expression plasmid showed a 2.3-fold increase, but this enhancement was almost completely abrogated by treatment with LY255283 (Fig. 1d, right panel). These results suggest that BLT2 mediates IL- 6 production in KRAS-mutant lung cancer cells.

To determine whether inhibition of the BLT2 cascade affects the proliferation of A549 and SK-LU-1 cells, we treated cells with
MK886 to inhibit 5 -LOX ${ }^{28,29}$ or baicalein to inhibit $12-$ LOX $^{30,31}$. Inhibition of 5-LOX, 12-LOX or BLT2 clearly suppressed the proliferation of A549 and SK-LU-1 cells (Fig. 1e). Similarly, when BEAS-2B cells were transfected with the KrasG12D expression plasmid, cell proliferation was strongly enhanced, but this enhancement was almost completely attenuated by inhibition of 5-/12-LOX or BLT2 (Fig. 1f).

\section{Increased expression of BLT2 cascade molecules in a mouse} model of KrasG12D-driven lung cancer

To examine the effect of BLT2 signaling on nodule progression in KRAS-mutant lung cancer in vivo, we used a transgenic mouse model with a lung-specific expression of mutant KRAS (Kras ${ }^{\mathrm{G} 12 \mathrm{D}}$ mice). In this model, mutant KrasG12D expression is driven by the promoter of human surfactant protein C (hSP-C) (Fig. 2a, top), which is expressed only in the lung (Fig. 2a, bottom right). In the $\mathrm{Kras}^{\mathrm{G} 12 \mathrm{D}}$ mice, nodules were observed only in the lungs from 8 weeks after birth (Fig. 2b). Then, we assessed the basal levels of p-CPLA $2,5-L O X, 12-L O X$, and BLT2 in lung tissue lysates. As shown in Fig. $2 c$, the levels of p-CPLA $2,5-L O X, 12-L O X$, and BLT2 were strongly enhanced in the lung tissues of the Kras ${ }^{\mathrm{G} 12 \mathrm{D}}$ mice compared with those of the WT mice. These results suggest that the expression of members of the $\mathrm{p}-\mathrm{CPLA}_{2}-5-/ 12-\mathrm{LOX}-\mathrm{BLT} 2$ cascade is upregulated in the KrasG12D-driven lung cancer mouse model. 
a
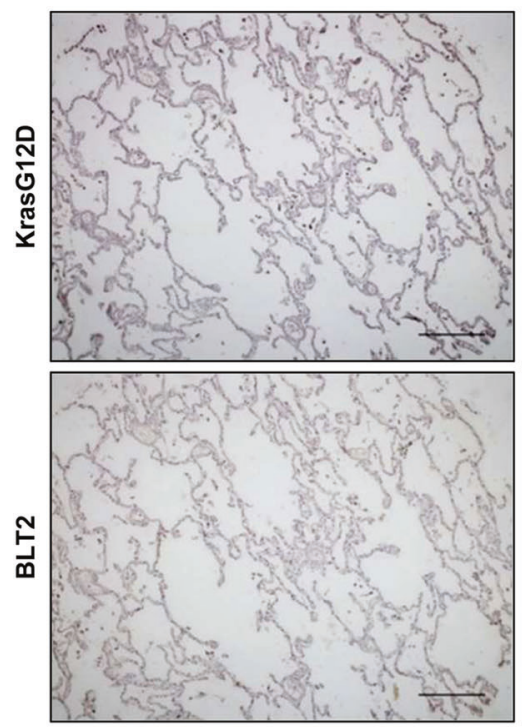

b
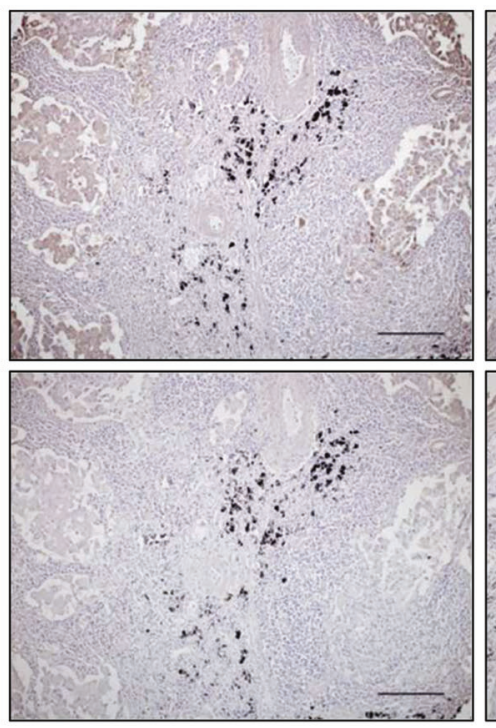

C

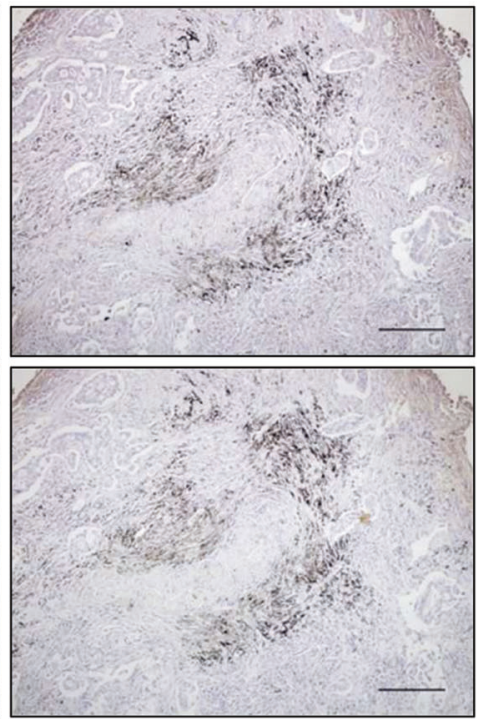

d

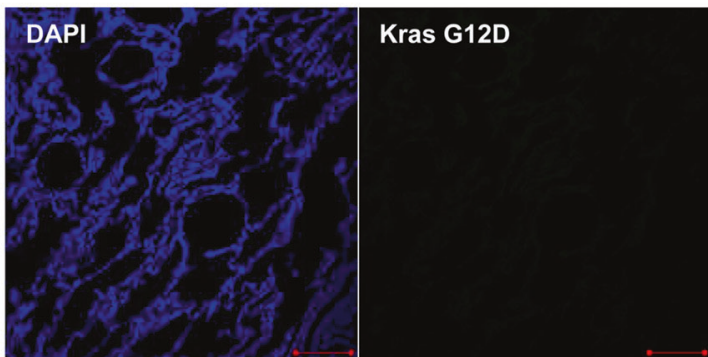

BLT2
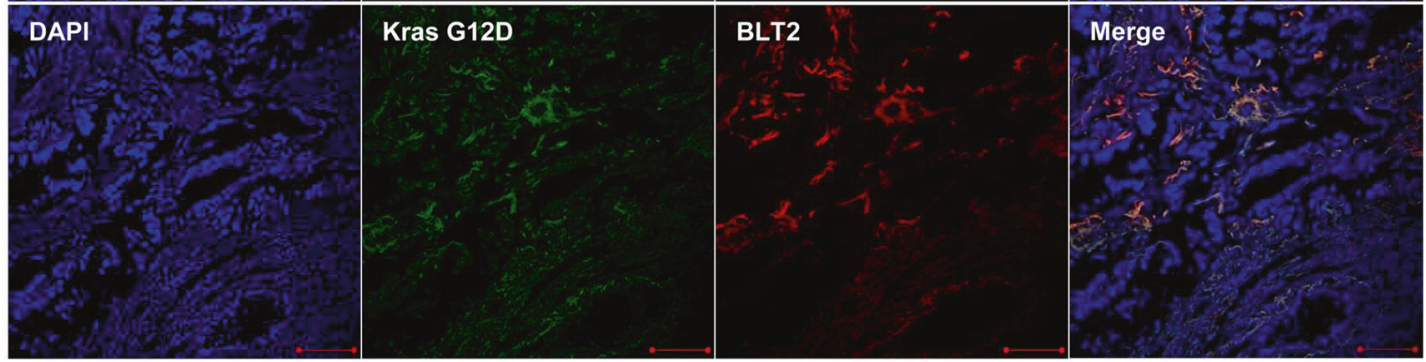

Fig. 5 High expression levels of BLT2 in lung adenocarcinoma patients with KRAS mutations. a-c IHC analysis of KrasG12D and BLT2 expression in lung adenocarcinoma patients (a normal lung tissue; b, c lung adenocarcinoma tissue). Scale bars, $400 \mu \mathrm{m}$. d IF analysis of KrasG12D and BLT2 expression in lung adenocarcinoma patients (upper panel, normal lung tissue; lower panel, lung adenocarcinoma tissue). Scale bars, $100 \mu \mathrm{m}$.

\section{Inhibition of 5-/12-LOX or BLT2 suppresses IL-6 production and KrasG12D-driven lung nodule formation}

Lung nodules were observed beginning at 8 weeks after birth in the $\mathrm{Kras}^{\mathrm{G} 12 \mathrm{D}}$ mice. To examine the suppressive effects of BLT2 cascade inhibition on KrasG12D-driven lung nodule formation, we injected inhibitors into the peritoneal cavity of the mice starting 9 weeks after birth once a week for 12 weeks (Fig. 3a). Interestingly, the number of lung nodules was significantly decreased by 5- or 12-LOX inhibitor treatment (Fig. 3b, c, e), and the elevated IL- 6 production in lung tissue was almost completely suppressed (Fig. 3f). We also investigated whether BLT2 inhibition could suppress tumor formation and IL- 6 production in these mice and observed that treatment with LY255283 clearly suppressed KRAS-driven lung tumor formation (Fig. $3 \mathrm{~g}-\mathrm{j}$ ) and IL-6 production (Fig. 3k).

Our analysis also showed that airway cellular infiltration, which represents lung airway inflammation, was suppressed by inhibitors of 5-LOX, 12-LOX, or BLT2 (Fig. 3d, i). Furthermore, we found that treatment with LY255283 effectively decreased the levels of
$\mathrm{p}-\mathrm{IKBa}$, an indicator of NF-KB activation, in mouse lung tissue (Fig. 3I). These results are consistent with previous results demonstrating that the BLT2 pathway stimulates the production of inflammatory cytokines via the transcription factor $\mathrm{NF}-\mathrm{KB}^{32}$. Moreover, the LY255283 treatment significantly reduced the levels of inflammatory cytokines and chemokines associated with the progression of lung cancer ${ }^{33-39}$ (IL-1 $\beta$, TNFa, CXCL1, GM-CSF, MUC4, and MUC5AC; Fig. 3m). Taken together, these results suggest that the BLT2 cascade modulates the synthesis of various proinflammatory agents, including IL-6, that are critical for KRASdriven lung tumor formation.

\section{KrasG12D-driven lung tumor formation and lung inflammation are attenuated in Blt2 KO mice}

To further determine whether BLT2 is involved in KRAS-driven lung tumor formation, we established a Kras ${ }^{\mathrm{G} 12 \mathrm{D}} / \mathrm{BLT} 2-\mathrm{KO}$ doublemutant mouse model. The Kras ${ }^{\mathrm{G} 12 \mathrm{D}} / \mathrm{BLT} 2-\mathrm{KO}$ mice had fewer lung nodules than the Kras ${ }^{\text {G12D }}$ mice (Fig. $4 a$, b, e). The BLT2 expression levels in the lung tissues of these double-mutant mice were lower 
Cohort: Pan-Lung Cancer database (TCGA, Nat Genet 2016, $n=1144$ )

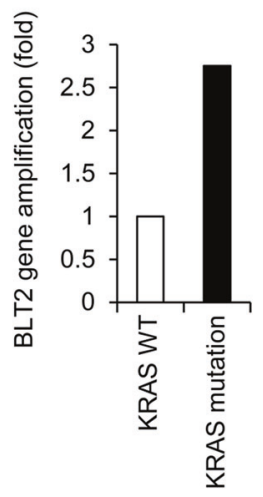

Fig. 6 KRAS-mutant lung cancer patients show elevated BLT2 gene amplification. a Fold increased BLT2 gene amplification in KRAS-mutant lung cancer patients compared with KRAS WT lung cancer patients. The Pan-Lung Cancer database (TCGA, Nat Genet 2016) cohort of 1144 lung cancer patients was analyzed by cBioPortal. b A model summarizing the findings from our study.

than those in the lung tissues of the $\operatorname{Kras}^{\mathrm{G} 12 \mathrm{D}}$ mice (Fig. $4 \mathrm{f}$ ). Furthermore, lung airway inflammation (Fig. 4c) and the number of immune cells in BALF (Fig. $4 \mathrm{~d}, \mathrm{~g}$ ) were substantially reduced in the Kras ${ }^{\mathrm{G} 12 \mathrm{D}} / \mathrm{BLT} 2-\mathrm{KO}$ mice compared with the Kras ${ }^{\mathrm{G} 12 \mathrm{D}}$ mice. The levels of $\mathrm{LTB}_{4}$ were also reduced in the $\mathrm{Kras}^{\mathrm{G}}{ }^{2 \mathrm{D}} / \mathrm{BLT} 2-\mathrm{KO}$ mice (Fig. 4h), consistent with the feedback regulatory mechanism between eicosanoid receptors and their ligands ${ }^{40,41}$. IL-6 expression was also reduced in the Kras ${ }^{\mathrm{G} 12 \mathrm{D}} / \mathrm{BLT} 2-\mathrm{KO}$ mice compared with the Kras ${ }^{\mathrm{G} 12 \mathrm{D}}$ mice (Fig. 4i). In addition, the IL-6 level did not differ between the KRAS ${ }^{\mathrm{WT}} / \mathrm{BLT} 2-\mathrm{KO}$ mice and the WT mice, suggesting that the effect of BLT2 KO is specific to KRAS-induced IL-6 production (Fig. 4j). Together, these results suggest that Blt2 KO effectively suppresses KrasG12D-driven lung inflammation and tumor formation.

\section{High BLT2 expression is observed in lung cancer patients with the KrasG12D mutation}

Next, we further examined the link between BLT2 expression and KrasG12D status in lung cancer patients. Thus, to determine whether BLT2 expression is increased in patients with KrasG12Dexpressing lung cancer, we performed IHC staining of paraffinembedded tissue sections derived from 45 patients with malignant lung adenocarcinoma. The expression of KrasG12D and BLT2 was not detected in normal human lung tissue (Fig. 5a). In contrast, the lung adenocarcinoma tissue sections showed positive staining for KrasG12D, and the KrasG12D-stained samples showed high BLT2 expression in the KrasG12D-positive region, consistent with the suggested role of elevated BLT2 expression in KRAS-driven lung cancer (Fig. 5b, c). To further analyze the role of elevated BLT2 expression in KRAS-driven lung cancer, we performed a co-staining experiment in lung tissues by IF analysis. As shown in Fig. $5 \mathrm{~d}$, we observed a clear colocalization of KrasG12D and BLT2 expression (Fig. 5d). Moreover, to further demonstrate the linkage between BLT2 expression and KRAS mutation in human lung cancer, we analyzed the patient database stratified by KRAS mutation. We used the Pan-Lung Cancer (TCGA, Nat Genet 2016, $n=1144$ ) database to analyze the specificity of KRAS mutation and BLT2. We found that the KRAS-mutant lung cancer patients showed 2.7-fold increased BLT2 gene amplification compared with the KRAS WT lung cancer patients (Fig. 6a). Together, our results indicate that BLT2 has a potential role in KRAS-driven lung cancer (the proposed signaling model is summarized in Fig. $6 \mathrm{~b}$ ) and that inhibition of the BLT2 cascade may be a new therapeutic strategy against KRAS-driven lung cancer.

\section{DISCUSSION}

In the present study, we demonstrated that the BLT2 cascade is critical for the progression of KRAS-driven lung cancer. Treatment with BLT2 cascade inhibitors clearly suppressed IL-6 production and lung tumor nodule formation in a KrasG12D-driven lung cancer mouse model. The contributory role of BLT2 in the proliferation of KRAS-driven lung tumors was further demonstrated in the Kras ${ }^{\mathrm{G} 12 \mathrm{D}} / \mathrm{BLT} 2 \mathrm{KO}$ model. These double-mutant mice showed clear reductions in the level of IL- 6 and the number of lung nodules compared with the $\mathrm{Kras}^{\mathrm{G} 12 \mathrm{D} / \mathrm{BLT} 2 \mathrm{WT} \text { mice. In }}$ support of the contributory role of BLT2 in KRAS-driven lung cancer progression, IHC analysis of tissues obtained from patients with KrasG12D mutant malignant lung adenocarcinoma showed high levels of BLT2 expression.

Lung cancer is currently considered a difficult-to-treat disease. More than half of lung cancer patients have KRAS or EGFR mutations ${ }^{42}$; however, there is no available effective cancer therapy for patients with KRAS-mutant lung cancer, in contrast to those with EGFR-mutant lung cancer. Patients with EGFRmutant lung cancer are commonly sensitive to receptor tyrosine kinase inhibitor (RTKi) drugs, which show some ability to improve overall survival ${ }^{43,44}$. However, patients with KRAS-mutant lung cancer are not sensitive to RTKi drugs; thus, an effective alternative treatment is urgently needed. Mutations in codons 12, 13, and 61 of KRAS have oncogenic potential, and KrasG12X mutations are the most abundant mutation types in lung cancer patients ${ }^{45}$. Among these mutations, KrasG12D is found in $\sim 15 \%$ of KRASmutant lung cancer cases, and more than half of never-smoking lung cancer patients have the KrasG12D mutation ${ }^{46}$. Based on these clinical observations, we used a mouse model with a lungspecific expression of the KrasG12D mutation to test BLT2 as an alternative target for KRAS-driven lung cancer. In this lung cancer model, inhibition of the BLT2 cascade effectively attenuated lung tumor progression.

A link between the KrasG12D mutation and BLT2 expression in lung cancer was also suggested by IHC and IF analysis of tissues derived from patients with malignant lung adenocarcinoma (Fig. 5). These KrasG12D-positive samples showed highly elevated BLT2 expression, in agreement with the contributory role of BLT2 in KRAS-driven human lung cancer. Of course, further experiments with more KRAS mutant lung cancer patient samples are needed to demonstrate the linkage between BLT2 expression and KRAS mutation in human lung cancer. The mechanism by which the expression levels of BLT2 and its ligand-producing enzymes (5LOX and 12-LOX) were highly increased by mutant KRAS is not clear, but we speculate that p38 kinase is involved. Previous studies have demonstrated that p38 kinase stimulates the phosphorylation of $\mathrm{CPLA}_{2}$, thus activating the $\mathrm{CPLA}_{2}-5-/ 12-\mathrm{LOX}$ cascade $^{47,48}$. Indeed, we observed that the KrasG12D mutant stimulated p38 kinase activity in the lung tissues of a KRAS-driven lung cancer mouse model (Supplementary Fig. 2a). In addition, the protein expression levels of 5-/12-LOX and BLT2 in SK-LU-1 cells were diminished by treatment with a p38 inhibitor (Supplementary Fig. 2b).

Inflammatory signaling plays an important role in cancer progression. In particular, inflammatory lipid mediators such as eicosanoids and their receptors have been suggested to establish a tumor-friendly environment by acting as local mediators. For example, cyclooxygenase (COX)-2 belongs to the proinflammatory eicosanoid family and increases lung cancer progression and lung inflammation ${ }^{49,50}$. In contrast, the roles of LOX- or LOX-derived lipid mediators in lung cancer, especially KRAS-driven lung cancer, remain to be elucidated. In this study, we demonstrated that the 
5-/12-LOX-BLT2 cascade is highly amplified during KRAS-driven lung cancer progression (Fig. 2c). The expression level of BLT2 was highly elevated in KRAS-mutant lung cancer cells and in both mouse and human lung cancers with KrasG12D mutations. We speculate that $\mathrm{LTB}_{4}$, a product of 5 -LOX, or 12(S)-HETE, a product of 12-LOX, interacts with its receptor, BLT2, to establish or mediate the tumor-promoting environment ${ }^{51}$. In addition, we found that the inhibition of BLT2 reduces the levels of its ligand-producing enzymes and ligands (Fig. $4 \mathrm{~h}$ and Supplementary Fig. 3), and this feedback regulation is frequently observed in eicosanoid receptors ${ }^{40,41}$. In this tumor-friendly environment, BLT2-mediated autocrine positive feedback loops could lead to more aggressive amplification of tumor progression ${ }^{52-54}$. In conclusion, to our knowledge, this report is the first to define the role of BLT2 in KRAS-driven lung cancer. We anticipate that these new findings will facilitate the development of strategies against lung cancer, especially KRAS-mutant lung cancer.

\section{REFERENCES}

1. Siegel, R. L., Miller, K. D. \& Jemal, A. Cancer statistics, 2020. CA Cancer J. Clin. 70, 7-30 (2020).

2. Collisson, E. A. et al. Comprehensive molecular profiling of lung adenocarcinoma. Nature 511, 543-550 (2014).

3. Ostrem, J. M., Peters, U., Sos, M. L., Wells, J. A. \& Shokat, K. M. K-Ras(G12C) inhibitors allosterically control GTP affinity and effector interactions. Nature $\mathbf{5 0 3}$ 548-551 (2013)

4. Park, J. et al. A Ras destabilizer KYA1797K overcomes the resistance of EGFR tyrosine kinase inhibitor in KRAS-mutated non-small cell lung cancer. Sci. Rep. 9, 648 (2019).

5. Downward, J. RAS's cloak of invincibility slips at last? Cancer Cell 25, 5-6 (2014).

6. Khan, N. \& Mukhtar, H. Dietary agents for prevention and treatment of lung cancer. Cancer Lett. 359, 155-164 (2015).

7. Caetano, M. S. et al. IL6 Blockade reprograms the lung tumor microenvironment to limit the development and progression of K-ras-mutant lung cancer. Cancer Res. 76, 3189-3199 (2016).

8. Yang, Y. et al. MicroRNA-218 functions as a tumor suppressor in lung cancer by targeting IL-6/STAT3 and negatively correlates with poor prognosis. Mol. Cancer 16, 141 (2017).

9. Liu, F. et al. Elevated IL-6/IL-1Ra ratio as a prognostic biomarker of poor chemotherapy efficacy in Chinese patients with metastatic NSCLC, validated in a Caucasian patient cohort. Mol. Clin. Oncol. 10, 309-317 (2019).

10. Silva, E. M. et al. High systemic IL-6 is associated with worse prognosis in patients with non-small cell lung cancer. PLoS ONE 12, e0181125 (2017).

11. Kang, S., Tanaka, T., Narazaki, M. \& Kishimoto, T. Targeting interleukin- 6 signaling in clinic. Immunity 50, 1007-1023 (2019).

12. Passiglia, F. et al. Treatment of advanced non-small-cell lung cancer: the 2019 AIOM (Italian Association of Medical Oncology) clinical practice guidelines. Crit. Rev. Oncol. Hematol. 146, 102858 (2019).

13. Yokomizo, T., Kato, K., Terawaki, K., Izumi, T. \& Shimizu, T. A second leukotriene B (4) receptor, BLT2. A new therapeutic target in inflammation and immunological disorders. J. Exp. Med. 192, 421-432 (2000).

14. Hennig, R. et al. BLT2 is expressed in PanINs, IPMNs, pancreatic cancer and stimulates tumour cell proliferation. Br. J. Cancer 99, 1064-1073 (2008).

15. Pace, E. et al. Cigarette smoke increases BLT2 receptor functions in bronchial epithelial cells: in vitro and ex vivo evidence. Immunology 139, 245-255 (2013).

16. Jala, V. R. et al. The Yin and Yang of leukotriene B4 mediated inflammation in cancer. Semin. Immunol. 33, 58-64 (2017).

17. Lee, A. J., Ro, M., Cho, K. J. \& Kim, J. H. Lipopolysaccharide/TLR4 stimulates IL-13 production through a MyD88-BLT2-linked cascade in mast cells, potentially contributing to the allergic response. J. Immunol. 199, 409-417 (2017).

18. Ro, M., Lee, A. J. \& Kim, J. H. 5-/12-Lipoxygenase-linked cascade contributes to the IL-33-induced synthesis of IL-13 in mast cells, thus promoting asthma development. Allergy 73, 350-360 (2018).

19. Radmark, O., Werz, O., Steinhilber, D. \& Samuelsson, B. 5-Lipoxygenase, a key enzyme for leukotriene biosynthesis in health and disease. Biochim. Biophys. Acta 1851, 331-339 (2015).

20. Tourdot, B. E. \& Holinstat, M. Targeting 12-lipoxygenase as a potential novel antiplatelet therapy. Trends Pharmacol. Sci. 38, 1006-1015 (2017).

21. Kwon, S. Y. \& Kim, J. H. Role of leukotriene B4 receptor-2 in mast cells in allergic airway inflammation. Int. J. Mol. Sci. 20, 2897 (2019).
22. Lee, S. et al. Melphalan inhibits adenoma development through modulating the expression of K-ras-specific markers in K-ras Tg mice. Int. J. Oncol. 37, 219-228 (2010).

23. Lee, A. J., Ro, M. \& Kim, J. H. Leukotriene B4 receptor 2 is critical for the synthesis of vascular endothelial growth factor in allergen-stimulated mast cells. J. Immunol. 197, 2069-2078 (2016).

24. Hall, Z. et al. Myc expression drives aberrant lipid metabolism in lung cancer. Cancer Res. 76, 4608-4618 (2016).

25. Brooks, G. D. et al. IL6 trans-signaling promotes KRAS-driven lung carcinogenesis. Cancer Res. 76, 866-876 (2016).

26. Wang, Y. C. et al. USP24 induces IL- 6 in tumor-associated microenvironment by stabilizing p300 and beta-TrCP and promotes cancer malignancy. Nat. Commun. 9, 3996 (2018).

27. Li, R., Huang, Y. \& Lin, J. Distinct effects of general anesthetics on lung metastasis mediated by IL-6/JAK/STAT3 pathway in mouse models. Nat. Commun. 11, 642 (2020).

28. Mayburd, A. L. et al. Ingenuity network-assisted transcription profiling: Identification of a new pharmacologic mechanism for MK886. Clin. Cancer Res. 12, 1820-1827 (2006).

29. Avis, I. et al. Inhibitors of the arachidonic acid pathway and peroxisome proliferator-activated receptor ligands have superadditive effects on lung cancer growth inhibition. Cancer Res. 65, 4181-4190 (2005).

30. Svensson Holm, A. C., Grenegård, M., Ollinger, K. \& Lindström, E. G. Inhibition of 12-lipoxygenase reduces platelet activation and prevents their mitogenic function. Platelets 25, 111-117 (2014).

31. Yarla, N. S. et al. Targeting arachidonic acid pathway by natural products for cancer prevention and therapy. Semin. Cancer Biol. 40-41, 48-81 (2016).

32. Nam, Y. H. et al. Leukotriene B4 receptor BLT-mediated phosphorylation of NFkappaB and CREB is involved in IL-8 production in human mast cells induced by Trichomonas vaginalis-derived secretory products. Microbes Infect. 13, 1211-1220 (2011).

33. Wu, C. et al. IL-1beta-mediated up-regulation of WT1D via miR-144-3p and their synergistic effect with NF-kappaB/COX-2/HIF-1alpha pathway on cell proliferation in LUAD. Cell. Physiol. Biochem. 48, 2493-2502 (2018).

34. Kalari, K. R. et al. Deep sequence analysis of non-small cell lung cancer: integrated analysis of gene expression, alternative splicing, and single nucleotide variations in lung adenocarcinomas with and without oncogenic KRAS mutations. Front. Oncol. 2, 12 (2012).

35. $\mathrm{Yu}, \mathrm{S}$. et al. $\mathrm{CXCL1}$ as an unfavorable prognosis factor negatively regulated by DACH1 in non-small cell lung cancer. Front. Oncol. 9, 1515 (2019).

36. Zhu, N. et al. Efficacy of granulocyte-macrophage colony-stimulating factor combined with metronomic paclitaxel in the treatment of Lewis lung carcinoma transplanted in mice. Oncotarget 9, 4951-4960 (2018).

37. Jonckheere, N. \& Van Seuningen, I. Integrative analysis of the cancer genome atlas and cancer cell lines encyclopedia large-scale genomic databases: MUC4/ MUC16/MUC20 signature is associated with poor survival in human carcinomas. J. Transl. Med. 16, 259 (2018).

38. Bauer, A. K. et al. Requirement for MUC5AC in KRAS-dependent lung carcinogenesis. JCI Insight 3, e120941 (2018).

39. Hocker, J. R. et al. Serum monitoring and phenotype identification of Stage I nonsmall cell lung cancer patients. Cancer Investig. 35, 573-585 (2017).

40. Tarighi, N. et al. Thromboxane-induced alpha-CGRP release from peripheral neurons is an essential positive feedback loop in capsaicin-induced neurogenic inflammation. J. Investig. Dermatol. 139, 656-664 (2019).

41. Madrigal-Martinez, A., Constancio, V., Lucio-Cazana, F. J. \& Fernandez-Martinez, A. B. PROSTAGLANDIN E2 stimulates cancer-related phenotypes in prostate cancer PC3 cells through cyclooxygenase-2. J. Cell. Physiol. 234, 7548-7559 (2019).

42. Salehi-Rad, R., Li, R., Paul, M. K., Dubinett, S. M. \& Liu, B. The biology of lung cancer: development of more effective methods for prevention, diagnosis, and treatment. Clin. Chest Med. 41, 25-38 (2020).

43. Herbst, R. S., Morgensztern, D. \& Boshoff, C. The biology and management of non-small cell lung cancer. Nature 553, 446-454 (2018).

44. Cheng, L. et al. Molecular pathology of lung cancer: key to personalized medicine. Mod. Pathol. 25, 347-369 (2012).

45. Lindsay, C. R., Jamal-Hanjani, M., Forster, M. \& Blackhall, F. KRAS: reasons for optimism in lung cancer. Eur. J. Cancer 99, 20-27 (2018).

46. $\mathrm{Yu}, \mathrm{H}$. A. et al. Prognostic impact of KRAS mutation subtypes in 677 patients with metastatic lung adenocarcinomas. J. Thorac. Oncol. 10, 431-437 (2015).

47. Cao, C. et al. Activation of CRHR1 contributes to cerebral endothelial barrier impairment via CPLA2 phosphorylation in experimental ischemic stroke. Cell. Signal. 66, 109467 (2020).

48. Korbecki, J., Baranowska-Bosiacka, I., Gutowska, I. \& Chlubek, D. The effect of reactive oxygen species on the synthesis of prostanoids from arachidonic acid. J. Physiol. Pharmacol. 64, 409-421 (2013). 
1568

49. Mantovani, A., Allavena, P., Sica, A. \& Balkwill, F. Cancer-related inflammation. Nature 454, 436-444 (2008).

50. Pan, Y. et al. Deletion of cyclooxygenase-2 inhibits K-ras-induced lung carcinogenesis. Oncotarget 6, 38816-38826 (2015)

51. Park, J. et al. BLT2, a leukotriene B4 receptor 2, as a novel prognostic biomarker of triple-negative breast cancer. BMB Rep. 51, 373-377 (2018).

52. Zhu, Z. et al. Inhibition of KRAS-driven tumorigenicity by interruption of an autocrine cytokine circuit. Cancer Discov. 4, 452-465 (2014).

53. Reynders, $\mathrm{N}$. et al. The distinct roles of $\mathrm{CXCR}_{3}$ variants and their ligands in the tumor microenvironment. Cells 8, 613 (2019).

54. Yang, Y. F. et al. A positive feedback loop of IL-17B-IL-17RB activates ERK/betacatenin to promote lung cancer metastasis. Cancer Lett. 422, 44-55 (2018).

\section{ACKNOWLEDGEMENTS}

This work was supported by a Bio and Medical Technology Development Program grant (2017M3A9D8063317) and a Mid-Career Researcher Program grant (2020R1A2B5B01002046) through the National Research Foundation (NRF) funded by the Ministry of Science, Information and Communication Technologies (ICT) and Future Planning of the Republic of Korea. This work was also supported by a Korean University Grant.

\section{COMPETING INTERESTS}

The authors declare no competing interests.

\section{ADDITIONAL INFORMATION}

Supplementary information The online version contains supplementary material available at https://doi.org/10.1038/s12276-021-00682-z.

Correspondence and requests for materials should be addressed to Jae-Hong Kim.

Reprints and permission information is available at http://www.nature.com/ reprints

Publisher's note Springer Nature remains neutral with regard to jurisdictional claims in published maps and institutional affiliations. cc) (i) Attribution 4.0 International License, which permits use adaptation, distribution and reproduction in any medium or format, as long as you give appropriate credit to the original author(s) and the source, provide a link to the Creative Commons license, and indicate if changes were made. The images or other third party material in this article are included in the article's Creative Commons license, unless indicated otherwise in a credit line to the material. If material is not included in the article's Creative Commons license and your intended use is not permitted by statutory regulation or exceeds the permitted use, you will need to obtain permission directly from the copyright holder. To view a copy of this license, visit http://creativecommons. org/licenses/by/4.0/.

(c) The Author(s) 2021 